\title{
Microarray expression analysis of MYCN-amplified neuroblastoma cells after inhibition of CDK2
}

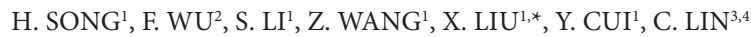 \\ ${ }^{1}$ Department of Neurosurgical Oncology, $1^{\text {st }}$ Hospital of Jilin University, Changchun, 130021 China; ${ }^{2}$ Department of Obstetrics and Gynecology, \\ $2^{\text {nd }}$ Hospital of Jilin University, Changchun, 130000 China; ${ }^{3}$ Neurological Research Center, the $1^{\text {st }}$ Hospital of Jilin University, Changchun, 130021 \\ China; ${ }^{4}$ Institute of Science and Technology, Brain-Inspired Intelligence, Fudan University, Shanghai, 200433 China
}

${ }^{*}$ Correspondence: liuxinr@jlu.edu.cn

Received July 18, 2016 / Accepted October 28, 2016

\begin{abstract}
The study aimed to explore the underlying molecular mechanisms of CDK2 inhibition in neuroblastoma by bioinformatics analysis. Gene expression profile GSE16480 was downloaded from the Gene Expression Omnibus. The differentially expressed genes (DEGs) were identified from IMR32 between each time point and average expression of all time points. Gene significance was calculated using dSVDsig algorithm of dnet package. Protein-protein interaction (PPI) network was built. Then, integrated with gene significance, a core PPI network was detected by dNetPipeline algorithm in dnet package. Finally, pathway enrichment analysis was performed for genes in network. Totally, 1524 DEGs were identified. CCNA2 (cyclin A2), EXO1 (exonuclease 1), RAD51AP1 (RAD51 associated protein 1), TOP2A (topoisomerase (DNA) II alpha) and $C D K 1$ (cyclin-dependent kinase 1) were selected as DEGs with higher connectivity after PPI network analysis. In the network, CCNA2, CDK1, BUB1B (BUB1 mitotic checkpoint serine/threonine kinase B) and CCNB1 (cyclin B1) were involved in cell cycle pathway. Additionally, CCNB1, CDK1, CCNE2 (Cyclin E2), and RRM2B (ribonucleotide reductase subunit $\mathrm{M} 2 \mathrm{~B}$ ) were involved in p53 signaling pathway. Cell cycle and p53 signaling pathway were closely associated with neuroblastoma after CDK2 inhibition. The DEGs, such as CCNA2, CCNB1, CDK1 and RRM2B may be the potential targets for neuroblastoma.
\end{abstract}

Key words: neuroblastoma, CDK2 inhibition, gene significance, bioinformatics

Neuroblastoma is the most common malignant pediatric tumor accounting for about $15 \%$ of cancer deaths in children [1]. Neuroblastomas are embryonic tumors originating from the transformation of neural crest progenitor cells [2]. More than half of patients subjected to neuroblastoma have metastases, leading to 5 -year survival rate of high-risk neuroblastoma patients below 35\% [3].

MYCN is the strongest genetic marker for treatment failure, being amplified in $25 \%$ of neuroblastoma tumors $[4,5]$. The MYCN proto-oncogene has been shown to regulate the expression of genes involved in many processes, including cell cycle and proliferation [6]. A variety of cell cycle regulating proteins in tumorigenesis has been identified, including cyclin-dependent kinases (CDK). A recent study has illustrated the importance of p21 in inhibiting CDK2 and restraining cell proliferation during development and differentiation [7]. Moreover, p21 is an vital target of p53 and regulates DNA- damage-induced cell-cycle arrest in G1 and G2 phases [8]. In consequence, CDK2 inhibition may induce apoptosis of neuroblastoma cells and be an effective therapy for childhood with MYCN-amplified neuroblastoma [9]. However, the specific underlying molecular mechanism associated with CDK2 inhibition and neuroblastoma is still largely unknown.

The microarray data GSE16480 [7] was generated and analyzed by Molenaar et al. Six most strongly regulated genes were identified by them and the study indicated that P53 pathway was involved in the CDK2 silencing-induced cell apoptosis. While, large information still remain in the mRNA expression profiles. In this study, we downloaded GSE16480 and identified the differentially expressed genes (DEGs) between each time point and average expression of all time points. Then, gene significance of DEGs were calculated and protein-protein interaction (PPI) network analysis were detected, followed by pathway enrichment analysis. 


\section{Materials and methods}

Microarray data. The microarray data GSE16480 [7] were obtained from Gene Expression Omnibus (GEO, http://www. ncbi.nlm.nih.gov/geo/) database based on the platform of Affymetrix Human Genome U133 Plus 2.0 Array. The dataset consisted of MYCN-amplified neuroblastoma cell IMR32 induced by CDK2 shRNA at 5 time points $(0,8,24,48$ and $72 \mathrm{~h}$ after transfection) in biological triplicate experiments.

Data preprocessing. The raw data in CEL files were preprocessed using AFFY package [10] in R software, following 4 steps including background correction by robust multi-array average (RMA), quantile normalization, probe summarization and $\log 2$ transformation of expression intensity. Then the expression value of probe level was obtained. The profiles in the probe set were averaged to get the expression value of each gene.

Identification of DEGs. DEGs from IMR32 between each time point and average expression of all time points were identified by Limma package in R [11]. Multiple testing was corrected by the Benjamini and Hochberg ( $\mathrm{BH})$ [12] to obtain the adjusted $\mathrm{p}$-value. The genes with adjusted $\mathrm{P}$-value $<0.05$ and $\mid \log 2 \mathrm{FC}$ (fold change) $\mid \geq 0.5$ were considered to be statistically significant. Then, those significant genes were analyzed using hierarchical clustering of the $\log 2$ values and displayed in a heatmap. Significant kyoto encyclopedia of genes and genomes (KEGG) pathway was plotted with plugin CyKEGGParser [13] in cytoscape.

Calculation of gene significance of DEGs. Singular value decomposition (SVD) is a factorization of a real or complex matrix, with many useful applications in statistics and signal processing. Gene significance was calculated using dSVDsig algorithm in dnet package [14] as follows: $A_{m \times n}$ $=S_{m x m} V_{m x n} D_{n x n} \cdot S_{m x m}$ and $D_{n x n}$ are unitary matrixes, $V_{m x n}$ is an augmented diagonal matrix, and the value on the diagonal of $V_{m x n}$ is the singular value of $A_{m \times n}$.

Detection of modules. Search Tool for the Retrieval of Interacting Genes (STRING) [15] is an online database which has been designed as a global perspective to evaluate PPI information. In this study, STRING was used to analyze the PPI network and only those with the threshold of combined score more than 0.9 screened from each time point were

Table 1. Pathways enriched by differentially expressed genes (DEGs) in the protein-protein interaction (PPI) network.

\begin{tabular}{lcc}
\hline Term & Count & P Value \\
\hline hsa04110:Cell cycle & 14 & $3.10 \mathrm{E}-14$ \\
hsa04114:Oocyte meiosis & 7 & $2.83 \mathrm{E}-05$ \\
hsa04914:Progesterone-mediated oocyte maturation & 6 & $1.06 \mathrm{E}-04$ \\
hsa04115:p53 signaling pathway & 5 & $5.40 \mathrm{E}-04$ \\
hsa03030:DNA replication & 4 & $1.05 \mathrm{E}-03$ \\
hsa00240:Pyrimidine metabolism & 5 & $1.90 \mathrm{E}-03$ \\
hsa03410:Base excision repair & 3 & $1.66 \mathrm{E}-02$ \\
\hline
\end{tabular}

Count: number of genes enriched in the term. selected as significant. Integrated with gene significance and PPI network, module with the maximum score was detected by dNetPipeline algorithm in dnet package [14].

Pathway enrichment analysis. Database for annotation, visualization, and integrated discovery (DAVID) [16] online tool was used in the present study to perform KEGG and Biocarta pathway enrichment analysis of DEGs in network. A $P$-value $<0.05$ was considered as statistically significant. In addition, significant pathways using plugin KEGGParser in cytoscape [17] were plotted to explore the important genes in neuroblastoma.

\section{Results}

Identification of DEGs. Totally, 1524 DEGs were screened from IMR32 between each time point and average expression of all time points. Specifically, 649 DEGs (411 up-regulated and 238 down-regulated) at T0, 499 DEGs (331 up-regulated and 168 down-regulated) at T8, 220 DEGs (192 up-regulated and 28 down-regulated) at T24, 398 DEGs (152 up-regulated and 246 down-regulated) at T48, and 1392 DEGs (552 up-regulated and 840 down-regulated) at T72 were identified. Hierarchical clustering revealed systematic variations in the expression of genes between DEGs at each time point group compared to the average expression of all time points group (Figure 1). The results demonstrated that there were two cases of these DEGs: the expression of genes was decreased or increased with increasing time. In addition, pathways enriched by DEGs in these two cases were shown in figure 2. Especially, both of DEGs in two modules were enriched in p53 signaling pathway. Thus, the distribution of DEGs in p53 signaling pathway was further studied, and function annotation revealed that down-regulated DEGs were mainly involved in cell cycle and up-regulated DEGs were mainly involved in apoptosis (Figure 3).

PPI network analysis. Based on the provided networks of gene significance and STRING, a new core PPI network of DEGs which included 103 nodes and 4393 edges with connectivity of 0.84 was constructed (Figure $4 \mathrm{~A}$ ). In this network, CCNA2 (cyclin A2), EXO1 (exonuclease 1), RAD51AP1 (RAD51 associated protein 1), TOP2A (topoisomerase (DNA) II alpha) and CDK1 (cyclin-dependent kinase 1) were selected as DEGs with higher connectivity. In addition, the time-changing expression trend of genes in network was plotted, and the results showed that time-changing of the majority of genes was relatively flat in the first 24 hours but decreased after 24 hours (Figure 4B). However, time-changing expression of 5 genes was different. They were $R R M 2 B$ (ribonucleotide reductase M2 B), MSH4 (mutS homolog 4), HIST1H2BD (histone cluster 1, $\mathrm{H} 2 \mathrm{bd}$ ), RET (ret proto-oncogene) and LUM (lumican).

Pathway enrichment analysis of DEGs in the network. KEGG and Biocarta pathway enrichment analysis showed that genes in PPI network were significantly enriched in cell cycle-related pathways, including Cell cycle, Oocyte meiosis, Cell Cycle: G2/M Checkpoint and p53 signaling pathways (Table 1 and Figure 5). In the network, CCNA2, CDK1, BUB1B 

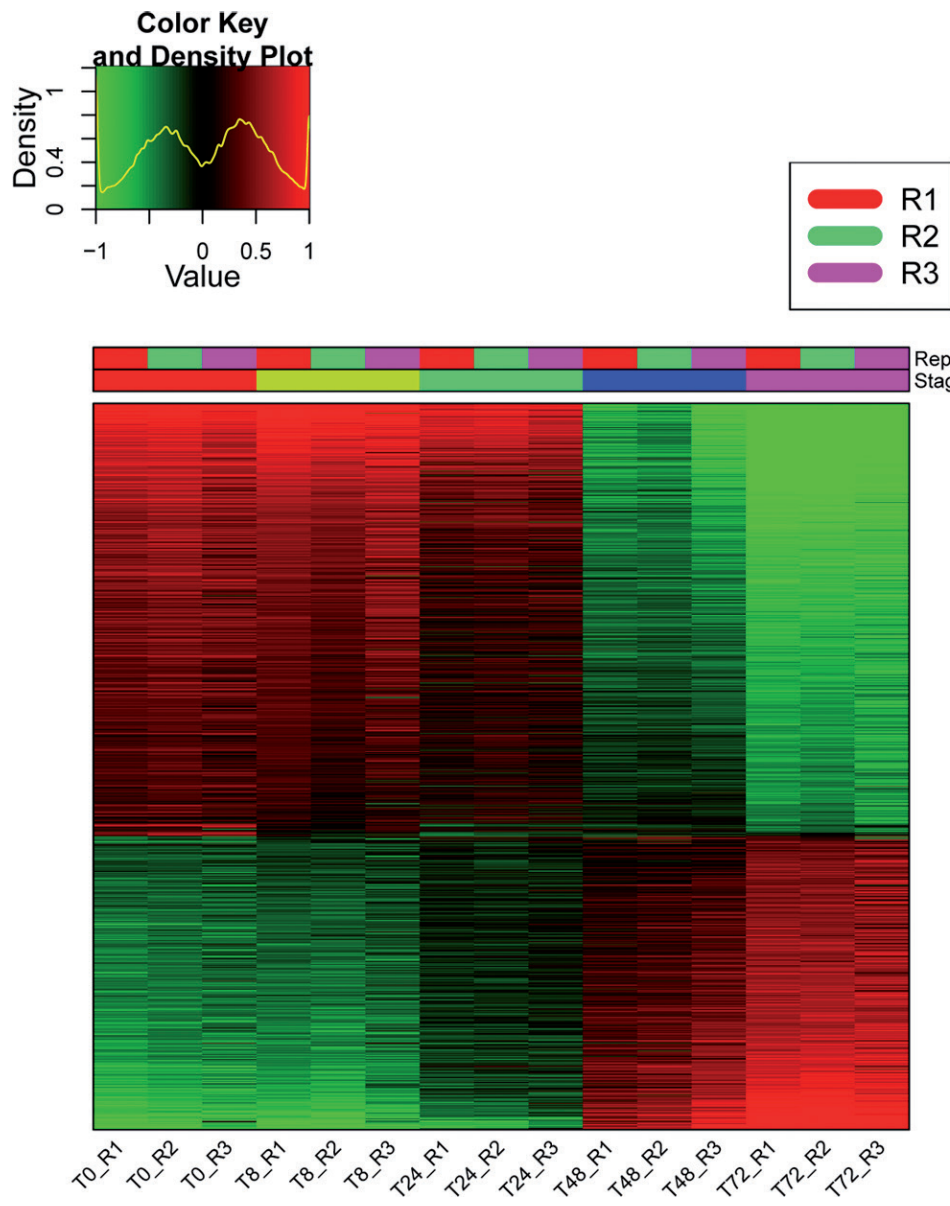

Replicates Stages

hsa03030:DNA replication

hsa04110: Cell cycle

hsa03430: Mismatch repair

hsa03440: Homologous recombination

hsa03420: Nucleotide excision repair

hsa03410:Base excision repair

hsa04114:Oocyte meiosis

hsa00230: Purine metabolism

hsa00240: Pyrimidine metabolism

hsa04914: Progesterone-mediated oocyte maturation

hsa04115:p53 signaling pathway

hsa00563: Glycosylphosphatidylinositol(GPI)-anchor biosynthesis

h_atrbrcaPathway:Role of BRCA1, BRCA2 and ATR in Cancer Susceptibility

h_DNAfragmentPathway:Apoptotic DNA fragmentation and tissue homeostasis

h_bard1Pathway:BRCA1-dependent Ub-ligase activity

h_g2Pathway:Cell Cycle: G2/M Checkpoint

h_cellcyclePathway:Cyclins and Cell Cycle Regulation

h_mcmPathway:CDK Regulation of DNA Replication

h_atmPathway:ATM Signaling Pathway

h_rbPathway:RB Tumor Suppressor/Checkpoint Signaling in response to DNA damage

h_ranMSpathway:Role of Ran in mitotic spindle regulation

h_aMANpathway:Steps in the Glycosylation of

Mammalian $\mathrm{N}$-linked Oligosaccarides

hsa04115:p53 signaling pathway

hsa05322:Systemic lupus erythematosus

hsa04360:Axon guidance

Figure 1. Hierarchical clustering heatmap of differentially expressed genes (DEGs) from neuroblatoma cells after inactivation of CDK2. Expression data are depicted as a data matrix where each row represents a gene and each column represents a sample. Expression levels are depicted according to the color scale shown at the top. Red and green indicate expression levels above and below the median, respectively. R1, R2 and R3 represent the time of replicates.
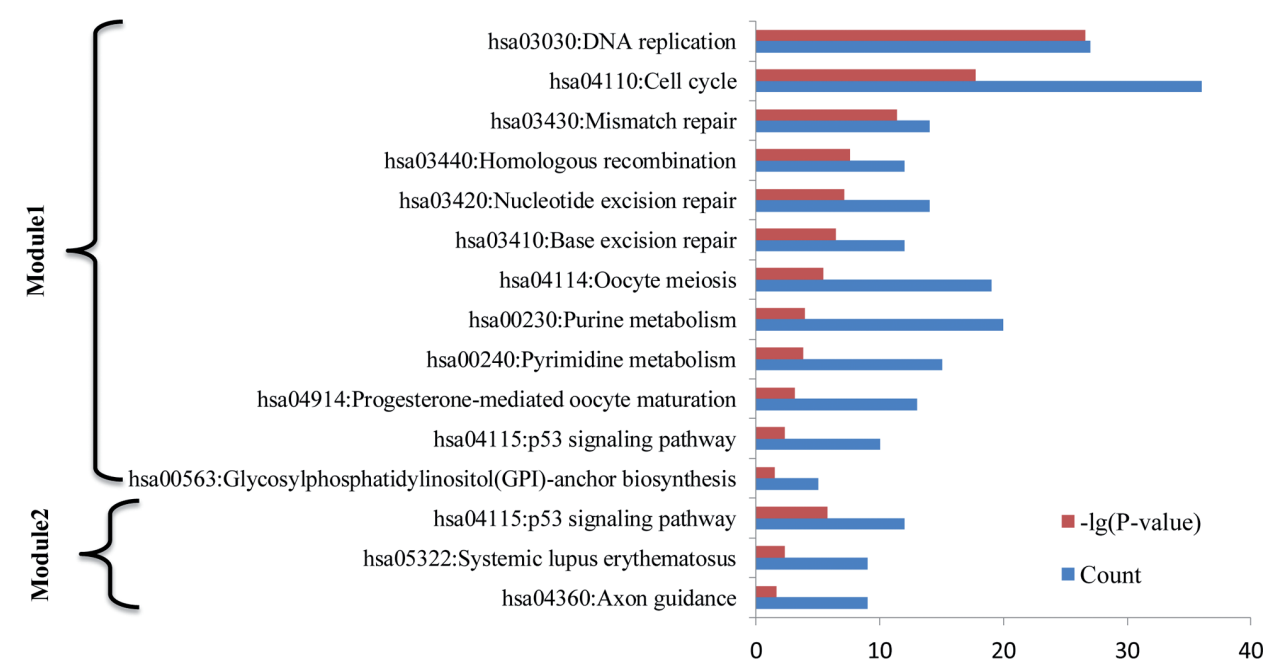

Figure 2. Pathways enriched by differentially expressed genes (DEGs) in two modules. Pathway terms were ranked by -lg(P-value) from top to bottom. Count indicates the number of genes enriched in the term. 


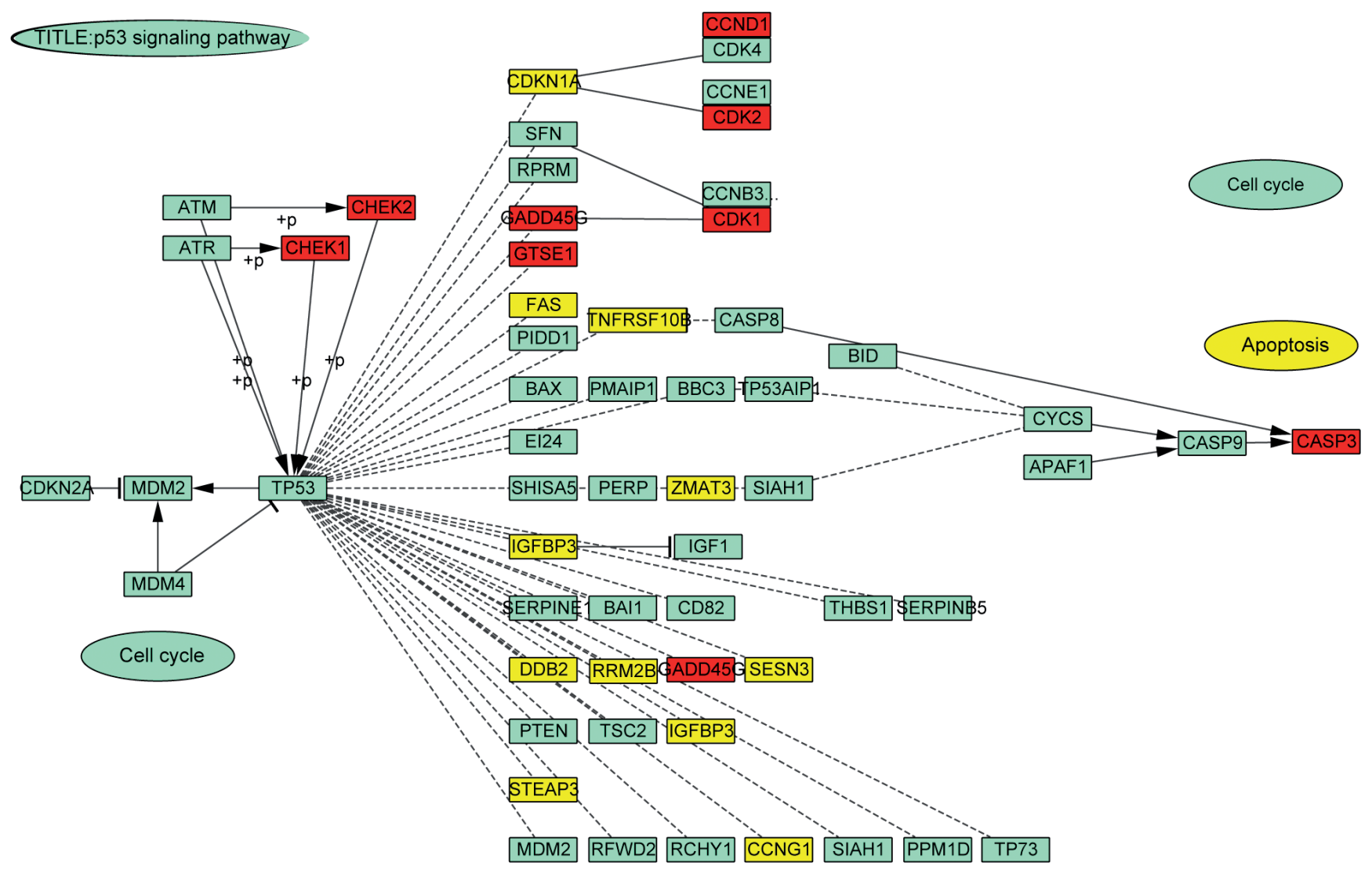

Figure 3. Distribution of differentially expressed genes (DEGs) in p53 signaling pathway. Red node represents down-regulated gene and yellow node represents up-regulated gene with increasing time. Green node represents genes without differential expression.

(BUB1 mitotic checkpoint serine/threonine kinase B) and CCNB1 (cyclin B1) were involved in pathway of cell cycle. Additionally, CCNB1, CDK1, CCNE2 (Cyclin E2), and RRM2B (ribonucleotide reductase subunit M2B) were involved in p53 signaling pathway.

\section{Discussion}

In this study, we found that after transfection with anti-CDK2 shRNA, a total of 1524 DEGs were screened from IMR32 cell line. In addition, in the PPI network analysis, CCNA2 and $C C N B 1$ were involved in cell cycle pathway. CCNB1, CDK1, $C C N E 2$ and $R R M 2 B$ were involved in p53 signaling pathway.

CDK2 inhibitor has been found synthetically lethal to MYNC over-expressing neuroblastoma cells [7]. In human cancer cells, CDK2 is an essential component of the cell cycle and with key function in tumorigenesis [18]. CDK2 in complex with Cyclin E is given a special importance since the complex sequentially phosphorylates retinoblastoma proteins $(\mathrm{Rb})$ on different threonine and serine residues which results in $\mathrm{Rb}$ inactivation required for transcriptional activation of genes in G1/S phase [19]. Once the cell passes restriction point of G1, CDK2 forms a complex with Cyclin A to carry out $S$ phase events [20]. In parallel, one level of regulation of CDK2-Cyclin complexes is provided by their bindings to $\mathrm{CDK}$ inhibitors.

CCNA2 and CCNB1 both belong to the highly conserved cyclin family that functions in cell proliferation and differentiation. The two cyclin members are essential for the control of the cell cycle at the checkpoint of G1/S and G2/M [21]. Recently, CCNA2 and CCNB1 have been found to interact with CDK1 that is important to drive the mammalian cell cycle [22]. Previous study has shown that CCNA2 has the ability to bind and activate $C D K 2$ [23]. Taken together, all of these may hint that CCNA2, and CCNB1 might be implicated in neuroblastoma through cell cycle pathway.

In addition, we constructed the PPI network with DEGs, performed pathway enrichment analysis of DEGs in the network, and found that the selected CDK1 and $R R M 2 B$ were involved in p53 signaling pathway. CDK1 is a member of the serine/threonine kinase family. The activity of $C D K 1$ plays a key role in the control of the eukaryotic cell cycle by modulating the centrosome cycle as well as mitotic onset; promoting G2-M transition, and regulating G1 progress and G1-S transition via association with multiple interphase cyclins [24]. In addition, $C D K 1$ inhibition has been found to induce cell death of neuroblastoma cells through the miR-34a-MYCN-survivin 

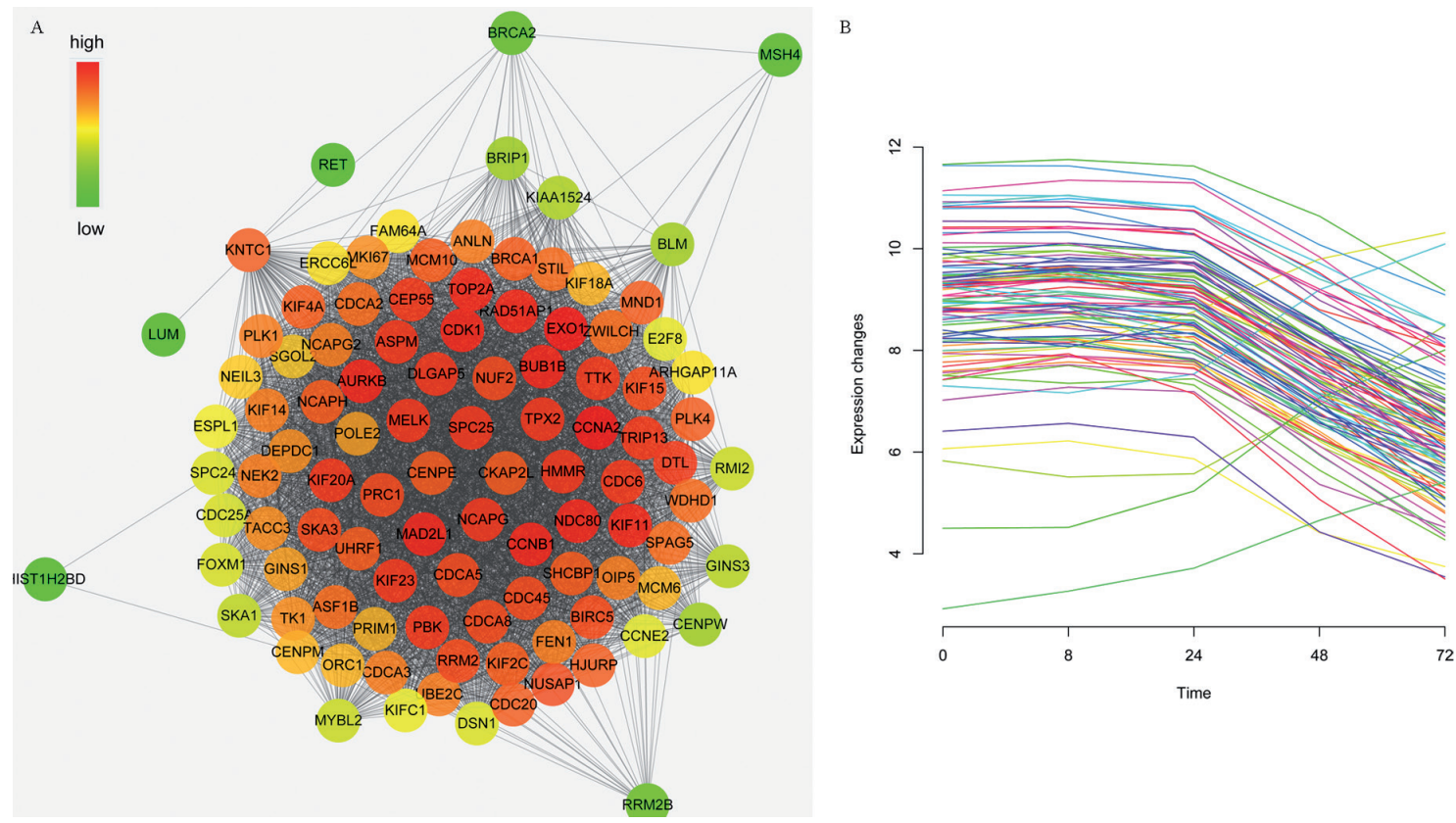

Figure 4. Protein interaction analysis of differentially expressed genes (DEGs). (A) A core protein-protein interaction network is detected. Color of node represents the size of the degree. (B) Time-changing expression trend of genes in network. The horizontal axis represents the time after transfection of small hairpin RNA. The vertical axis represents gene expression change.

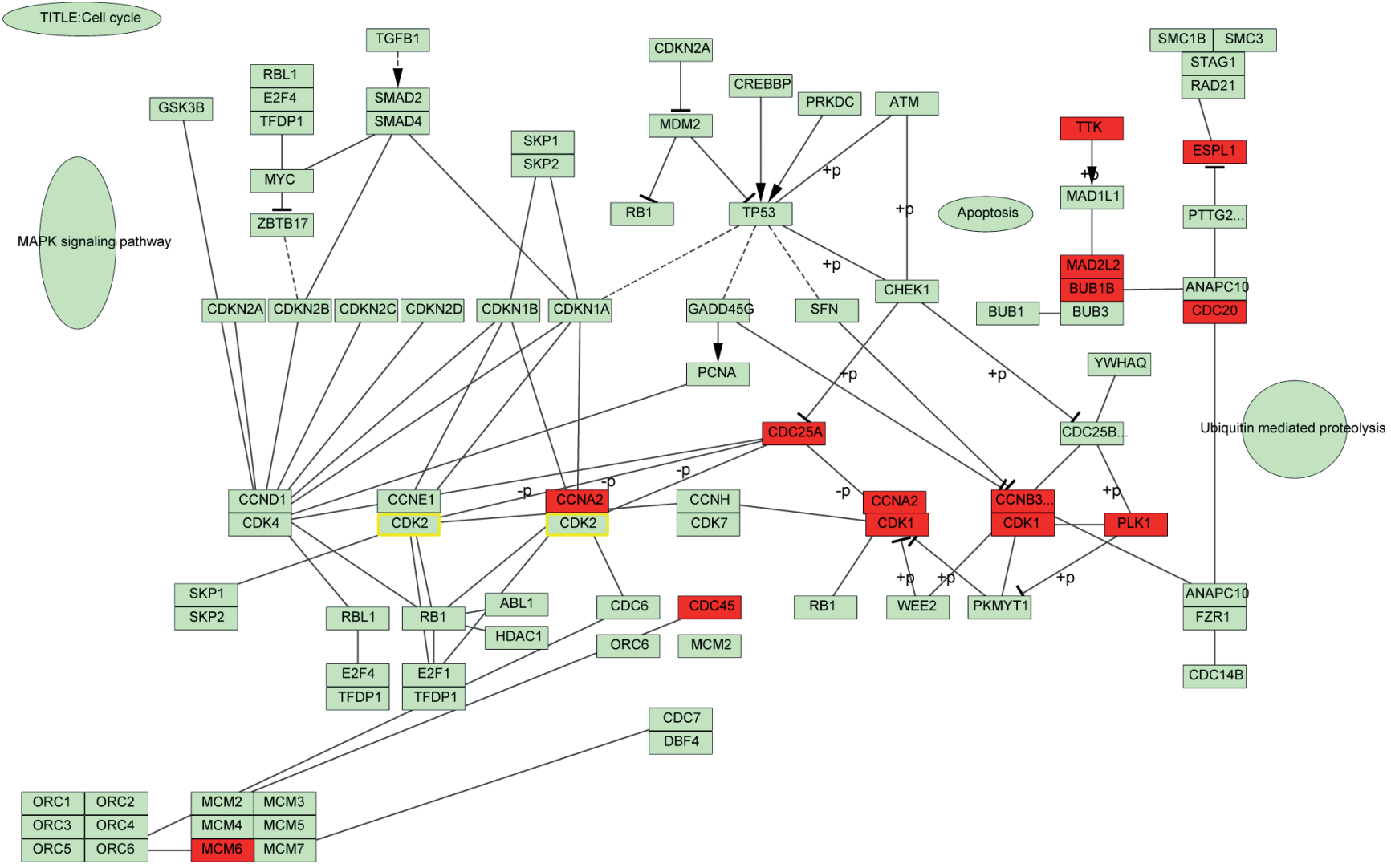

Figure 5. Distribution of genes of protein-protein interaction network in cell cycle pathway. The red nodes represent genes in network and highlight box node represents CDK2. 
pathway [25], suggesting that $C D K 1$ might play an important role in inhibiting neuroblastoma.

$R R M 2 B$, also known as p53R2, encodes a homologue of the R2 subunit of ribonucleotide reductase that forms a competent enzyme complex with the catalytic R1 subunit in nonproliferative cells [26]. p53R2 has recently been shown to function in nuclear DNA repair and to provide dNTPs for mitochondria DNA replication $[27,28]$. After DNA damage in response to irradiation or chemotherapeutic agents, p53 enhances the expression of p53R2 and induces cell cycle arrest in G1/G2 phases [29]. Additionally, p53R2 has been shown to up-regulate p21 and down-regulate cyclin D contributing to cell cycle arrest [30]. Moreover, p53R2 has dual opposite roles in before cancer development and after cancer formation [31]. Interestingly, p53R2 and Akt put each other in line with cancer development and progression [32]. Notably, the Akt pathway is activated in neuroblastoma cells and inhibition could decrease neuroblastoma tumor mass $[33,34]$. As mentioned above, up-regulated $R R M 2 B$ after CDK2 silencing may inhibit neuroblastoma progression.

In conclusion, we found that cell cycle and p53 signaling pathway were closely associated with neuroblastoma after CDK2 inhibition. Our data provide a comprehensive bioinformatics analysis of DEGs and pathways which may be involved in neuroblastoma after CDK2 inhibition. The findings in current study may contribute to our understanding of the underlying molecular mechanisms of neuroblastoma and the DEGs such as CCNA2, CCNB1, CDK1 and RRM2B have the potential to be used as targets for neuroblastoma diagnosis and treatment. However, more studies on improving diagnosis of neuroblastoma through regulating DEGs are still needed.

\section{References}

[1] MARIS JM, HOGARTY MD, BAGATELL R, COHN SL. Neuroblastoma. Lancet 2007; 369: 2106-2120. https://doi. org/10.1016/S0140-6736(07)60983-0

[2] SCHULTE JH, LINDNER S, BOHRER A, MAURER J, DE PRETER K et al. MYCN and ALKF1174L are sufficient to drive neuroblastoma development from neural crest progenitor cells. Oncogene 2013; 32: 1059-1065. https://doi.org/10.1038/ onc. 2012.106

[3] ZAGE PE, KLETZEL M, MURRAY K, MARCUS R, CASTLEBERRY R et al. Outcomes of the POG 9340/9341/9342 trials for children with high-risk neuroblastoma: A report from the Children's Oncology Group. Pediatr Blood Cancer 2008; 51: 747-753. https://doi.org/10.1002/pbc. 21713

[4] BOWEN KA, CHUNG DH. Recent advances in neuroblastoma. N Engl J Med 2010; 362: 27-36.

[5] DOMINGO-FERNANDEZ R, WATTERS K, PISKAREVA O, STALLINGS RL, BRAY I. The role of genetic and epigenetic alterations in neuroblastoma disease pathogenesis. Pediatr Surg Int 2013; 29: 101-119. https://doi.org/10.1007/s00383$\underline{012-3239-7}$
[6] GUSTAFSON WC, WEISS WA. Myc proteins as therapeutic targets. Oncogene 2010; 29: 1249-1259. https://doi. org/10.1038/onc.2009.512

[7] MOLENAAR JJ, EBUS ME, GEERTS D, KOSTER J, LAMERS $F$ et al. Inactivation of CDK2 is synthetically lethal to MYCN over-expressing cancer cells. Proc Natl Acad Sci U S A 2009; 106: 12968-12973. https://doi.org/10.1073/pnas.0901418106

[8] GARTEL AL, TYNER AL. Transcriptional regulation of the p21 (WAF1/CIP1) gene. Exp Cell Res 1999; 246: 280-289. https://doi.org/10.1006/excr.1998.4319

[9] GOGOLIN S, EHEMANN V, BECKER G, BRUECKNER L $M$, DREIDAX D et al. CDK4 inhibition restores $G_{1}-S$ arrest in MYCN-amplified neuroblastoma cells in the context of doxorubicin-induced DNA damage. Cell Cycle 2013; 12: 1091-1104. https://doi.org/10.4161/cc.24091

[10] GAUTIER L, COPE L, BOLSTAD BM, IRIZARRY RA. affy-analysis of Affymetrix GeneChip data at the probe level. Bioinformatics 2004; 20: 307-315. https://doi.org/10.1093/ bioinformatics/btg 405

[11] SMYTH G. limma: Linear Models for Microarray Data. p 397-420. In: R. Gentleman, V. Carey, W. Huber, R. Irizarry and S. Dudoit (Eds.), Bioinformatics and Computational Biology Solutions Using R and Bioconductor, Springer-Verlag New York, 2005, pp 474. 978-0-387-25146-2.

[12] BENJAMINI Y, HOCHBERG Y. Controlling the false discovery rate: a practical and powerful approach to multiple testing. J R Statist Soc B 1995; 57: 289-300.

[13] NERSISYAN L, SAMSONYAN R, ARAKELYAN A. CyKEGGParser: tailoring KEGG pathways to fit into systems biology analysis workflows. F1000Research 2014; 3: 145. https://doi. org/10.12688/f1000research.4410.2

[14] FANG H, GOUGH J. The ,dnet'approach promotes emerging research on cancer patient survival. Genome Med 2014; 6: 64 .

[15] FRANCESCHINI A, SZKLARCZYK D, FRANKILD S, KUHN M, SIMONOVIC M et al. STRING v9.1: proteinprotein interaction networks, with increased coverage and integration. Nucleic Acids Res 2013; 41: D808-815. https:// doi.org/10.1093/nar/gks1094

[16] DENNIS G JR, SHERMAN BT, HOSACK DA, YANG J, GAO W ET AL. DAVID: database for annotation, visualization, and integrated discovery. Genome Biol 2003; 4: P3. https://doi. org/10.1186/gb-2003-4-5-p3

[17] ARAKELYAN A, NERSISYAN L. KEGGParser: parsing and editing KEGG pathway maps in Matlab. Bioinformatics 2013; 29: 518-519. https://doi.org/10.1093/bioinformatics/bts730

[18] HARBOUR JW, DEAN DC. The Rb/E2F pathway: expanding roles and emerging paradigms. Genes Dev 2000; 14: 2393-2409. https://doi.org/10.1101/gad.813200

[19] MALUMBRES M, BARBACID M. Mammalian cyclindependent kinases. Trends Biochem Sci 2005; 30: 630-641. https://doi.org/10.1016/j.tibs.2005.09.005

[20] QIAO L, PAUL P, LEE S, QIAO J, WANG Y et al. Differential Regulation of Cyclin-Dependent Kinase Inhibitors in Neuroblastoma Cells. Biochem Biophys Res Commun 2013; 435: 295-299. https://doi.org/10.1016/j.bbrc.2013.04.023

[21] CHO RJ, HUANG M, CAMPBELL MJ, DONG H, STEINMETZ L et al. Transcriptional regulation and function during 
the human cell cycle. Nat Genet 2001; 27: 48-54. https://doi. org/10.1038/83751

[22] SANTAMARIA D, BARRIERE C, CERQUEIRA A, HUNT $\mathrm{S}$, TARDY C et al. Cdk1 is sufficient to drive the mammalian cell cycle. Nature 2007; 448: 811-815. https://doi.org/10.1038/ nature 06046

[23] NEBEN K, TEWS B, WROBEL G, HAHN M, KOKOCINSKI $\mathrm{F}$ et al. Gene expression patterns in acute myeloid leukemia correlate with centrosome aberrations and numerical chromosome changes. Oncogene 2004; 23: 2379-2384. https://doi. org/10.1038/sj.onc. 1207401

[24] MALUMBRES M, BARBACID M. Cell cycle, CDKs and cancer: a changing paradigm. Nat Rev Cancer 2009; 9: 153-166. https://doi.org/10.1038/nrc2602

[25] CHEN Y, TSAI YH, TSENG SH. Inhibition of cyclin-dependent kinase 1-induced cell death in neuroblastoma cells through the microRNA-34a-MYCN-survivin pathway. Surgery 2013; 153: 4-16. https://doi.org/10.1016/j.surg.2012.03.030

[26] THELANDER L. Ribonucleotide reductase and mitochondrial DNA synthesis. Nat Genet 2007; 39: 703-704. https:// doi.org/10.1038/ng0607-703

[27] PONTARIN G, FERRARO P, HAKANSSON P, THELANDER L, REICHARD P et al. p53R2-dependent ribonucleotide reduction provides deoxyribonucleotides in quiescent human fibroblasts in the absence of induced DNA damage. J Biol Chem 2007; 282: 16820-16828. https://doi.org/10.1074/jbc. M701310200

[28] BOURDON A, MINAI L, SERRE V, JAIS JP, SARZI E et al. Mutation of RRM2B, encoding p53-controlled ribonucleotide reductase (p53R2), causes severe mitochondrial DNA depletion. Nat Genet 2007; 39: 776-780. https://doi.org/10.1038/ ng2040

[29] NAKAMURA Y. Isolation of p53-target genes and their functional analysis. Cancer Sci 2004; 95: 7-11. https://doi. org/10.1111/j.1349-7006.2004.tb03163.x

[30] ZHANG K, WU J, WU X, WANG X, WANG Y et al. p53R2 inhibits the proliferation of human cancer cells in association with cell-cycle arrest. Mol Cancer Ther 2011; 10: 269-278. https://doi.org/10.1158/1535-7163.MCT-10-0728

[31] YOUSEFI B, RAHMATI M, AHMADI Y. The roles of p53R2 in cancer progression based on the new function of mutant p53 and cytoplasmic p21. Life Sci 2014; 99: 14-17. https://doi. org/10.1016/j.lfs.2014.01.063

[32] YOUSEFI B, SAMADI N, AHMADI Y. Akt and p53R2, partners that dictate the progression and invasiveness of cancer. DNA Repair (Amst) 2014; 22: 24-29. https://doi.org/10.1016/j. dnarep.2014.07.001

[33] FANG CX, YANG X, SREEJAYAN N, REN J. Acetaldehyde promotes rapamycin-dependent activation of p70 S6K and glucose uptake despite inhibition of Akt and mTOR in dopaminergic SH-SY5Y human neuroblastoma cells. Exp Neurol 2007; 203: 196-204. https://doi.org/10.1016/j.expneurol.2006.08.002

[34] CHESLER L, SCHLIEVE C, GOLDENBERG DD, KENNEY A, KIM G et al. Inhibition of phosphatidylinositol 3-kinase destabilizes Mycn protein and blocks malignant progression in neuroblastoma. Cancer Res 2006; 66: 8139-8146. https:// doi.org/10.1158/0008-5472.CAN-05-2769 